[Article]

\title{
中温固体氧化物燃料电池 $\mathrm{La}_{1.6} \mathrm{Sr}_{0.4} \mathrm{Ni}_{1-x} \mathrm{Cu}_{x} \mathrm{O}_{4}$ 阴极材料的制备及 电化学性能
}

\author{
李 强* 赵 辉 江 瑞 郭力帆 \\ (黑龙江大学化学化工与材料学院, 功能无机材料化学教育部重点实验室, 哈尔滨 150080)
}

\begin{abstract}
摘要: 采用甘氨酸-硝酸盐法合成了中温固体氧化物燃料电池阴极材料 $\mathrm{La}_{1.6} \mathrm{Sr}_{0.4} \mathrm{Ni}_{1-x} \mathrm{Cu}_{x} \mathrm{O}_{4}(x=0.2,0.4,0.6$, 0.8 ), 利用 X射线衍射(XRD)和扫描电子显微镜(SEM)对其结构和微观形貌进行了表征. 结果表明, 该阴极材料 与固体电解质 $\mathrm{Ce}_{0.9} \mathrm{Gd}_{0.1} \mathrm{O}_{1.95}(\mathrm{CGO})$ 在 $1000{ }^{\circ} \mathrm{C}$ 烧结时不发生化学反应, 且烧结 $4 \mathrm{~h}$ 后, 二者之间可形成良好的 接触界面. 利用电化学交流阻抗谱技术对阴极材料的电化学性能进行研究, 结果显示, 当 $\mathrm{Cu}$ 离子掺杂量 $(x)$ 为 0.6 时, $\mathrm{La}_{1.6} \mathrm{Sr}_{0.4} \mathrm{Ni}_{0.4} \mathrm{Cu}_{0.6} \mathrm{O}_{4}$ 阴极具有最小的极化电阻, 在空气中当测试温度为 $750{ }^{\circ} \mathrm{C}$ 时, 极化电阻为 $0.35 \Omega$. $\mathrm{cm}^{2}$. 在不同氧分压条件下电化学阻抗谱分析结果表明, 电极上的两个氧还原反应主要包含氧离子从三相界面 向电解质 CGO 转移的过程和电荷的迁移过程, 其中电荷的迁移过程为电极反应的速率控制步骤. $\mathrm{La}_{1.6} \mathrm{Sr}_{0.4} \mathrm{Ni}_{0.4} \mathrm{Cu}_{0.6} \mathrm{O}_{4}$ 电极在空气中 $700{ }^{\circ} \mathrm{C}$ 和阴极电流密度为 $45 \mathrm{~mA} \cdot \mathrm{cm}^{-2}$ 时, 阴极过电位为 $45 \mathrm{mV}$. 本研究的初 步结果表明 $\mathrm{La}_{1.6} \mathrm{Sr}_{0.4 \mathrm{Ni}_{1-} \mathrm{Cu}} \mathrm{Cu}_{4}$ 材料是一种电化学性能较为优良的新型中温固体氧化物燃料电池(IT-SOFC)阴 极材料.
\end{abstract}

关键词: 中温固体氧化物燃料电池; $\mathrm{La}_{1.6} \mathrm{Sr}_{0.4} \mathrm{Ni}_{1-x} \mathrm{Cu}_{\times} \mathrm{O}_{4}$ 阴极材料; 甘氨酸-硝酸盐法; 电化学性能; 氧还原反应

中图分类号: O646; TM911.4

\section{Synthesis and Electrochemical Properties of $\mathrm{La}_{1.6} \mathrm{Sr}_{0.4} \mathrm{Ni}_{1-x} \mathrm{Cu}_{x} \mathrm{O}_{4}$ as Cathode Materials for Intermediate Temperature Solid Oxide Fuel Cells}

\author{
LI Qiang* Z ZHAO Hui JIANG Rui GUO Li-Fan \\ (Key Laboratory of Functional Inorganic Material Chemistry, Ministry of Education, School of Chemistry and Materials Science, \\ Heilongjiang University, Harbin 150080, P. R. China)
}

\begin{abstract}
Cathodic materials $\mathrm{La}_{1.6} \mathrm{Sr}_{0.4} \mathrm{Ni}_{1-x} \mathrm{Cu}_{x} \mathrm{O}_{4}(x=0.2,0.4,0.6,0.8)$, for an intermediate temperature solid oxide fuel cell (IT-SOFC), were prepared by a glycine-nitrate process and characterized by X-ray diffraction (XRD), scanning electron microscopy (SEM). Results showed that no reaction occurred between the $\mathrm{La}_{1.6} \mathrm{Sr}_{0.4} \mathrm{Ni}_{1-x} \mathrm{Cu}_{x} \mathrm{O}_{4}$ electrode and the $\mathrm{Ce}_{0.9} \mathrm{Gd}_{0.1} \mathrm{O}_{1.95}$ (CGO) electrolyte at $1000{ }^{\circ} \mathrm{C}$, and that the electrode formed good contact with the electrolyte after sintering at $1000^{\circ} \mathrm{C}$ for $4 \mathrm{~h}$. Electrochemical AC impedance spectroscopy measurements were used to study cathodic performance. The $\mathrm{La}_{1.6} \mathrm{Sr}_{0.4} \mathrm{Ni}_{0.4} \mathrm{Cu}_{0.6} \mathrm{O}_{4}$ cathode gave the lowest polarization resistance $\left(R_{p}\right)$ of $0.35 \Omega \cdot \mathrm{cm}^{2}$ at $750{ }^{\circ} \mathrm{C}$ in air. Electrode properties of $\mathrm{La}_{1.6} \mathrm{Sr}_{0.4} \mathrm{Ni}_{1-x} \mathrm{Cu}_{x} \mathrm{O}_{4}$ were studied under various temperatures and oxygen partial pressures. The two main oxygen reduction processes at the cathode are the oxygen ion transfer from the triple phase boundary to CGO electrolyte, and the charge transfer process. Charge transfer is the major rate limiting step for $\mathrm{La}_{1.6} \mathrm{Sr}_{0.4} \mathrm{Ni}_{1-x} \mathrm{Cu}_{x} \mathrm{O}_{4}$ cathode. The $\mathrm{La}_{1.6} \mathrm{Sr}_{0.4} \mathrm{Ni}_{0.4} \mathrm{Cu}_{0.6} \mathrm{O}_{4}$ cathode exhibited the lowest overpotential, about 45 $\mathrm{mV}$ for a current density of $45 \mathrm{~mA} \cdot \mathrm{cm}^{-2}$ at $700{ }^{\circ} \mathrm{C}$ in air. This preliminary work showed that the present
\end{abstract}

Received: May 21, 2012; Revised: June 26, 2012; Published on Web: June 27, 2012.

"Corresponding author. Email: hematite1211@yahoo.com.cn; Tel: +86-451-86608426.

The project was supported by the Fund from Heilongjiang Educational Department, China (12521396).

黑龙江省教育厅(12521396)资助项目

(C) Editorial office of Acta Physico-Chimica Sinica 
$\mathrm{La}_{1.6} \mathrm{Sr}_{0.4} \mathrm{Ni}_{1-x} \mathrm{Cu}_{x} \mathrm{O}_{4}$ materials may be potential cathodes for use in IT-SOFCs.

Key Words: Intermediate temperature solid oxide fuel cell; $\quad \mathrm{La}_{1.6} \mathrm{Sr}_{0.4} \mathrm{Ni}_{1-x} \mathrm{Cu}_{x} \mathrm{O}_{4}$ cathode material; Glycine-nitrate process; Electrochemical property; Oxygen reduction reaction

\section{1 引 言}

近年来, 一些具有氧离子-电子混合传导的类钲 钛矿型 $\mathrm{A}_{2} \mathrm{BO}_{4}$ 混合导体氧化物成为人们研究的热点 材料. 这类材料属于 $\mathrm{K}_{2} \mathrm{NiF}_{4}$ 结构, 是由 $\mathrm{ABO}_{3}$ 钙铁矿 结构层与 $\mathrm{AO}$ 岩盐层相互交叠而成的层状化合物, 由于具有独特的晶体结构、良好的电化学性能以及 较高的化学稳定性与催化活性, 在新材料开发方面 已得到了高度的重视. ${ }^{1-4}$ 有关这类材料的晶体结构、 磁性和气体催化性能的报道较多, 而将其用于固体 氧化物燃料电池电极方面的研究却相对较少. ${ }^{5-7}$ 同 传统的钙钛矿型 $\mathrm{ABO}_{3}$ 氧化物 $\mathrm{La}_{1-x} \mathrm{Sr}_{x} \mathrm{MnO}_{3}$ (LSM) 和 $\mathrm{Ba}_{0.5} \mathrm{Sr}_{0.5} \mathrm{Co}_{1-x} \mathrm{Fe}_{x} \mathrm{O}_{3}(\mathrm{BSCF})$ 电极材料相比较, 这类 材料在化学相容性、电化学性能以及氧的扩散和表 面交换能力等方面显示了明显的优势, 这些都说明 $\mathrm{A}_{2} \mathrm{BO}_{4}$ 型氧化物是一种潜在的中温固体氧化物燃料 电池(IT-SOFC)阴极材料. . $^{811}$

有关类钙钛矿型镍酸盐氧化物的性能研究已 经有过一些报道, 它们显示了较高的混合电导率及 良好的氧输运能力. $\mathrm{Al}$ Daroukh 等 ${ }^{2}$ 报道了 $\mathrm{A}_{2} \mathrm{BO}_{4}$ 型 镍酸盐的混合电导率和热膨胀系数(TEC), 结果表 明, 这类氧化物比 $\mathrm{ABO}_{3}$ 型氧化物具有更好的热稳 定性, 同时这些镍酸盐氧化物的 TEC 与传统的电解 质材料 YSZ ( $8 \%$ 钎稳定化氧化锆) 和 $\mathrm{Ce}_{0.9} \mathrm{Gd}_{0.1} \mathrm{O}_{1.95}$ (CGO) 具有很好的热匹配性. Jorgensen 等 ${ }^{13}$ 发现 $\mathrm{La}_{2-x} \mathrm{Sr}_{x} \mathrm{NiO}_{4}$ 材料有较高的氧扩散系数和表面交换 系数, 并且在较宽的温度和氧分压范围内具有很 高的氧透过性, 是一类性能较佳的透氧膜材料. 在 以前的研究中也发现 $\mathrm{La}_{2-x} \mathrm{Sr}_{x} \mathrm{NiO}_{4}$ 氧化物作为阴极 材料有较佳的电化学性能与热稳定性能, 其中, $\mathrm{La}_{1.6} \mathrm{Sr}_{0.4} \mathrm{NiO}_{4}$ 材料在 $\mathrm{La}_{2-x} \mathrm{Sr}_{x} \mathrm{NiO}_{4}$ 体系中具有最小的 极化电阻和最高的电导率数值, 然而其中温极化电 阻却相对较大..$^{14,15}$ 以前的研究 ${ }^{16,17}$ 发现, 在 $\mathrm{ABO}_{3}$ 或 $\mathrm{A}_{2} \mathrm{BO}_{4}$ 型复合氧化物 $\mathrm{B}$ 位掺杂 $\mathrm{Cu}$ 元素可以提高材 料的氧离子输运性能及增加材料的氧空位浓度, 这 对于改善固体氧化物燃料电池阴极材料的电化学 性能是极为有利的. 因此, 本文采用甘氨酸-硝酸盐 法制备了 $\mathrm{Cu}$ 掺杂的 $\mathrm{La}_{1.6} \mathrm{Sr}_{0.4} \mathrm{NiO}_{4}$ 材料, 并研究了电 极材料的电化学性能和电极上的氧还原反应动力
学, 考察其作为中温固体氧化物燃料电池阴极材料 的可行性.

\section{2 实验部分}

\section{1 电极材料的制备}

$\mathrm{La}_{1.6} \mathrm{Sr}_{0.4} \mathrm{Ni}_{1-x} \mathrm{Cu}_{x} \mathrm{O}_{4}$ 材料采用甘氨酸(Gly)-硝酸盐 法制备: 按 $n_{\mathrm{La}}: n_{\mathrm{Sr}}: n_{\mathrm{N}}: n_{\mathrm{Cu}}=1.6: 0.4:(1-x): x \quad(x=0.2,0.4$, $0.6,0.8$ ) 的化学计量比准确移取硝酸镧(分析纯, 上 海精科试剂研究所)、硝酸锶(分析纯, 北京亚太龙兴 化工有限公司)、硝酸镍和硝酸铜(分析纯, 天津市科 密欧化学试剂公司)的水溶液, 混合. 再按甘氨酸与 溶液中金属离子物质的量比 $\left(n_{\mathrm{Gly}}:\left(n_{\mathrm{La}}+n_{\mathrm{Sr}}+n_{\mathrm{Ni}}+n_{\mathrm{Cu}}\right)\right)=2:$ 1 的比例, 加入计量甘氨酸(分析纯, 天津市科密欧 化学试剂公司), 搅拌使其完全溶解, 以使其与金属 离子形成配合物. 快速加热浓缩混合液, 直至剧烈燃 烧, 产生粉末状的氧化物. 将粉末研磨后, 于 $800{ }^{\circ} \mathrm{C}$ 进行烧结; 继续研磨粉末, 然后将其在 $1100{ }^{\circ} \mathrm{C}$ 下烧 结 $10 \mathrm{~h}$, 得到产物. 产物相纯度以及化学稳定性通过 粉末 $X$ 射线衍射 $(X R D)$ 进行表征.

\section{2 电极的制备}

取适量的 $\mathrm{La}_{1.6} \mathrm{Sr}_{0.4} \mathrm{Ni}_{1-x} \mathrm{Cu}_{x} \mathrm{O}_{4}$ 粉末，加入含 $3 \%$ (w) 乙基纤维素的松油醇(贵研铂业有限公司)溶液, 进行研磨, 得到的浆状物均匀地涂在 $\mathrm{Ce}_{0.9} \mathrm{Gd}_{0.1} \mathrm{O}_{1.95}$ 陶瓷片的一侧作为工作电极, $\mathrm{Pt}$ 参比电极制备于工 作电极的同一侧, 在另一侧均匀地涂上金浆(贵研铂 业有限公司) 作为对电极, 然后在空气中 900$1100{ }^{\circ} \mathrm{C}$ 烧结 $4 \mathrm{~h}$, 得到测试电池. $\mathrm{Ce}_{0.9} \mathrm{Gd}_{0.1} \mathrm{O}_{1.95}$ 粉末 制备见文献. ${ }^{18} \mathrm{CGO}$ 陶瓷片由粉末在 $220 \mathrm{MPa}$ 下加 压成型, 再于 $1400{ }^{\circ} \mathrm{C}$ 烧结 $12 \mathrm{~h}$ 得到. 工作电极与对 电极上面均铺有 Pt 集流网 (贵研铂业有限公司), 并 通过 $\mathrm{Pt}$ 丝(贵研铂业有限公司)与电化学仪器相联, 电极的微观结构用扫描电镜(SEM) (日本日立 Hitachi S-4700)进行观察.

\section{3 材料的结构及电化学性能表征}

合成的粉料采用德国布鲁克 D8-Advance 型 X 射线粉末衍射仪进行物相分析, 工作电流 $10 \mathrm{~mA}$, 管 电压 $40 \mathrm{kV}, \mathrm{Cu} K_{a}$ 靶辐射. 电极的电化学性能用交流 阻抗技术进行研究(荷兰Autolab PGStat 30 电化学 
工作站), 扫描频率范围为 $1 \mathrm{MHz}-0.01 \mathrm{~Hz}$, 测试条 件为空气-氮气混合气氛, 温度为 $500-750{ }^{\circ} \mathrm{C}$. 阴极 极化曲线的测定通过电压阶梯扫描得到. 阴极上的 极化过电位通过下式计算: $\eta_{\mathrm{WE}}=\Delta U_{\mathrm{WR}}-i R_{\mathrm{el}}$, 其中 $\eta_{\mathrm{WE}}$ 为阴极过电位, $\Delta U_{\mathrm{wR}}$ 为工作电极与参比电极的电位 差, $i$ 为流经电池的电流, $R_{\mathrm{el}}$ 为电解质电阻.

\section{3 结果与讨论}

图 1(a) 为 $\mathrm{La}_{1.6} \mathrm{Sr}_{0.4} \mathrm{Ni}_{1-x} \mathrm{Cu}_{x} \mathrm{O}_{4}(x=0.2,0.4,0.6$, $0.8)$ 系列氧化物粉体的 XRD 谱图. 从图可看出, 形成 的物相均为单一的类钙钛矿结构, 没有发现其它杂 相生成. 随着 $\mathrm{Cu}$ 离子取代量的增加, 衍射角度发生 小的偏移, 但未从根本上改变材料的晶体结构. 晶 胞体积略有增大, 引起晶体结构畸变, 但这种畸变 效应没有 $\mathrm{Sr}$ 掺杂时明显. ${ }^{19,20}$ 为考察电极与电解质材 料的高温相容性, 选取阴极 $\mathrm{La}_{1.6} \mathrm{Sr}_{0.4} \mathrm{Ni}_{0.4} \mathrm{Cu}_{0.6} \mathrm{O}_{4}$ 材料 与电解质 $\mathrm{CGO}$ 粉末按 $1: 1$ 质量比进行混合, 在 $1100{ }^{\circ} \mathrm{C}$ 下烧结 $12 \mathrm{~h}$, 从烧结后的 XRD 谱(图 1(b)) 可 以看出, 只有 $\mathrm{La}_{1.6} \mathrm{Sr}_{0.4} \mathrm{Ni}_{0.4} \mathrm{Cu}_{0.6} \mathrm{O}_{4}$ 与 $\mathrm{CGO}$ 的特征衍射
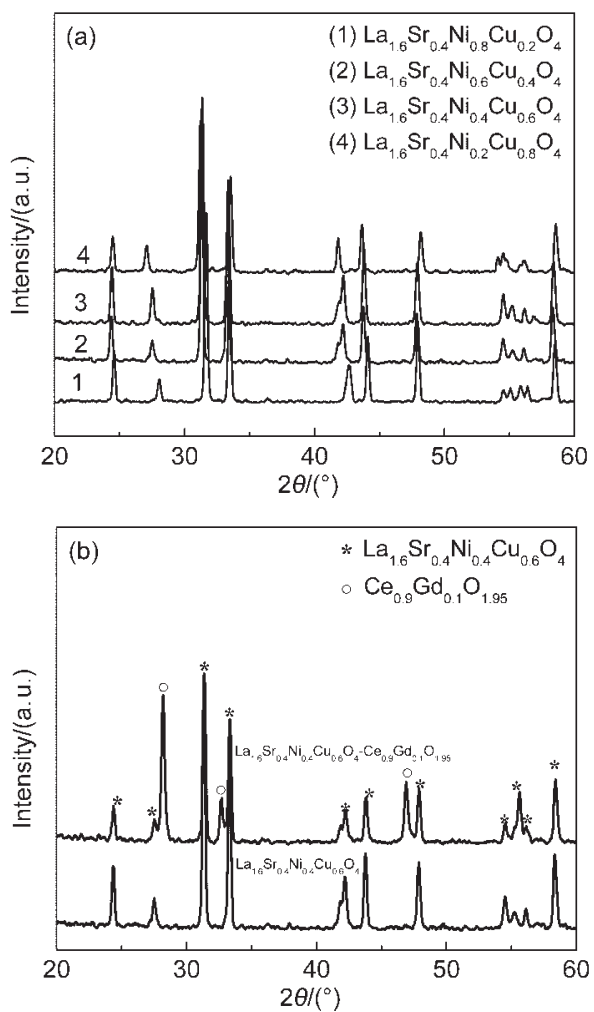

图 $1 \mathrm{La}_{1.6} \mathrm{Sr}_{0.4} \mathrm{Ni}_{1-x} \mathrm{Cu}_{x} \mathrm{O}_{4}(x=0.2,0.4,0.6,0.8)$ 粉体(a)和 $\mathrm{La}_{1.6} \mathrm{Sr}_{0.4} \mathrm{Ni}_{0.4} \mathrm{Cu}_{0.6} \mathrm{O}_{4}-\mathrm{Ce}_{0.9} \mathrm{Gd}_{0.1} \mathrm{O}_{1.95}$ 粉体(b)在 $1100{ }^{\circ} \mathrm{C}$ 空气中 烧结 $12 \mathrm{~h}$ 后的 XRD图

Fig.1 XRD patterns of the $\mathrm{La}_{1.6} \mathrm{Sr}_{0.4} \mathrm{Ni}_{1-x} \mathrm{Cu}_{x} \mathrm{O}_{4}(x=0.2,0.4$, 0.6, 0.8) powders (a) and $\mathrm{La}_{1.6} \mathrm{Sr}_{0.4} \mathrm{Ni}_{0.4} \mathrm{Cu}_{0.6} \mathrm{O}_{4}-\mathrm{Ce}_{0.9} \mathrm{Gd}_{0.1} \mathrm{O}_{1.95}$ powders (b) after sintering at $1100{ }^{\circ} \mathrm{C}$ for $12 \mathrm{~h}$ in air
峰, 没有其它的杂质峰出现. 这样可以确定两种材 料在 $1100{ }^{\circ} \mathrm{C}$ 范围内不会发生化学反应，二者之间 有较好的高温化学相容性.

为了考察电极的烧结条件对其电化学性能的 影响, 测试了在不同温度下烧结 $4 \mathrm{~h}$ 所得到的 $\mathrm{La}_{1.6} \mathrm{Sr}_{0.4} \mathrm{Ni}_{0.4} \mathrm{Cu}_{0.6} \mathrm{O}_{4}$ 电极在空气中 $750{ }^{\circ} \mathrm{C}$ 条件下的阻 抗谱, 如图 2 所示. 可以看出, 经过 $900{ }^{\circ} \mathrm{C}$ 烧结的电 极, 其极化电阻相对较大. 这可能是由于较低的烧 结温度不能使电极粒子之间以及电极粒子与电解 质界面形成良好的接触, 粒子之间相互独立, 连结 不够充分, 从而导致界面电阻的增加. 当烧结温度 为 $1000{ }^{\circ} \mathrm{C}$ 时, 电极的极化电阻达到最小值. 而烧结 温度升高到 $1100{ }^{\circ} \mathrm{C}$ 后, 电极的极化电阻又进一步 增大, 这主要是由于因烧结温度过高, 易长成大粒 子, 这样就会减少氧气的扩散通道, 使氧气的气相 输运过程受阻, 影响氧离子的及时生成, 即导致界 面电阻的增加. 类似的现象在其它 $\mathrm{A}_{2} \mathrm{BO}_{4}$ 型阴极材 料研究体系也有所发现. ${ }^{21,22}$ 图 3(a) 和 3(b) 分别为 $1000{ }^{\circ} \mathrm{C}$ 烧结的 $\mathrm{La}_{1.6} \mathrm{Sr}_{0.4} \mathrm{Ni}_{0.4} \mathrm{Cu}_{0.6} \mathrm{O}_{4}$ 电极表面及横断 面 SEM 图像. 可以看出, 电极表面粒子尺寸约为 $100 \mathrm{~nm}$, 粒子之间有一定的烧结连接, 形成较好的 多孔结构. 电极与电解质形成较好的接触界面, 电 极平均厚度约为 $15 \mu \mathrm{m}$. 根据以上结果, 可以确定 电极在 CGO 固体电解质上最佳的烧结温度为 $1000{ }^{\circ} \mathrm{C}$

图 4 为 $\mathrm{La}_{1.6} \mathrm{Sr}_{0.4} \mathrm{Ni}_{1-x} \mathrm{Cu}_{x} \mathrm{O}_{4}(x=0.2,0.4,0.6,0.8)$ 电 极的极化电阻随温度变化的 Arrhenius 曲线. 从图中 可以看到, 随着 $\mathrm{Cu}$ 掺杂量 $(0.2 \leq x \leq 0.6)$ 的增加, 电极 的极化电阻逐渐降低, 但当掺杂量增大到 0.8 时, 极

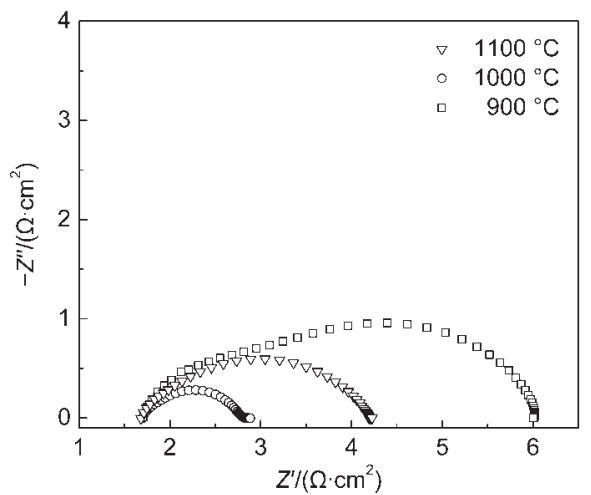

图 2 在不同温度下烧结的 $\mathrm{La}_{1.6} \mathrm{Sr}_{0.4} \mathrm{Ni}_{0.4} \mathrm{Cu}_{0.6} \mathrm{O}_{4}$ 电极在 $750{ }^{\circ} \mathrm{C}$ 空气中测得的阻抗谱

Fig.2 Impedance spectra of $\mathrm{La}_{1.6} \mathrm{Sr}_{0.4} \mathrm{Ni}_{0.4} \mathrm{Cu}_{0.6} \mathrm{O}_{4}$ electrode sintered at different temperatures for $4 \mathrm{~h}$ and then measured at $750{ }^{\circ} \mathrm{C}$ in air 

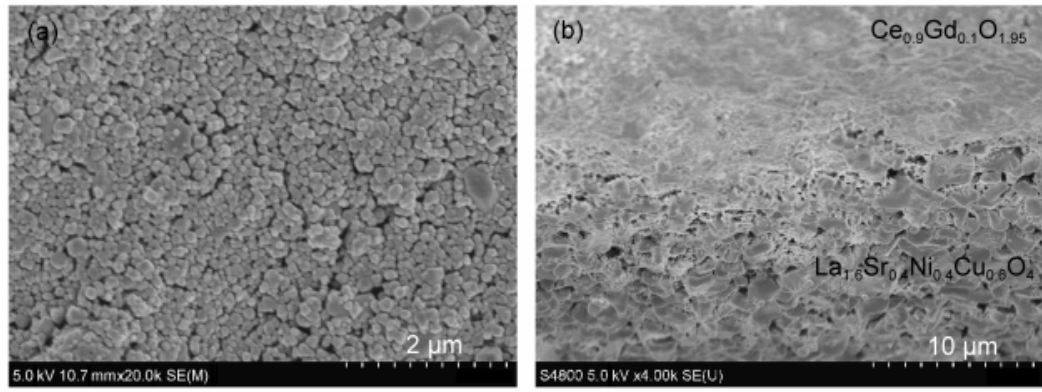

图 $31000{ }^{\circ} \mathrm{C}$ 烧结后的的 $\mathrm{La}_{1.6} \mathrm{Sr}_{0.4} \mathrm{Ni}_{0.4} \mathrm{Cu}_{0.6} \mathrm{O}_{4}$ 电极表面(a)及电极和电解质横断面(b)的 $\mathrm{SEM}$ 图

Fig.3 Surface (a) and cross-section (b) SEM images of the $\mathrm{La}_{1.6} \mathrm{Sr}_{0.4} \mathrm{Ni}_{0.4} \mathrm{Cu}_{0.6} \mathrm{O}_{4}$ electrode sintered at $1000{ }^{\circ} \mathrm{C}$

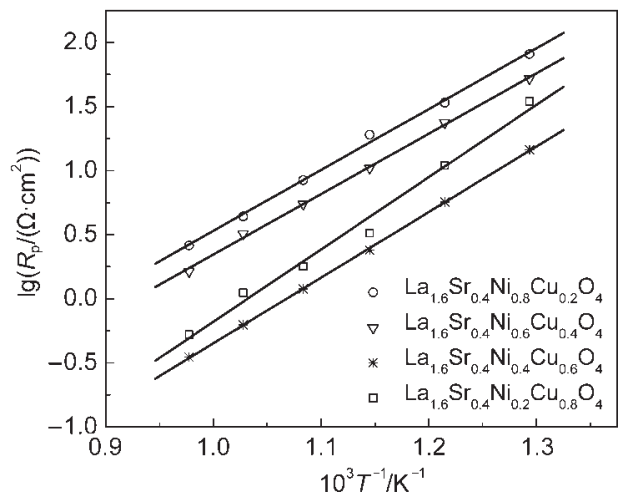

图 4 在空气中 $\mathrm{La}_{1.6} \mathrm{Sr}_{0.4} \mathrm{Ni}_{1-x} \mathrm{Cu}_{x} \mathrm{O}_{4}$ 电极极化电阻的 Arrhenius 图

Fig.4 Arrhenius plots of the polarization resistances of $\mathrm{La}_{1.6} \mathbf{S r}_{0.4} \mathrm{Ni}_{1-x} \mathrm{Cu}_{x} \mathrm{O}_{4}$ electrodes under air

化电阻又开始增加. 当 $\mathrm{Cu}$ 的掺杂量为 0.6 时, $\mathrm{La}_{1.6} \mathrm{Sr}_{0.4} \mathrm{Ni}_{0.4} \mathrm{Cu}_{0.6} \mathrm{O}_{4}$ 电极的极化电阻达到最小值. 这 可能是由于 $\mathrm{Cu}$ 的掺杂导致体系中氧缺陷浓度发生 了变化, 增大了电极材料的氧空位浓度, 从而改善 了电极的电催化活性. 当测试温度为 $750{ }^{\circ} \mathrm{C}$ 时 $\mathrm{La}_{1.6} \mathrm{Sr}_{0.4} \mathrm{Ni}_{0.4} \mathrm{Cu}_{0.6} \mathrm{O}_{4}$ 电极的极化电阻为 $0.35 \Omega \cdot \mathrm{cm}^{2}$, 这一数值同文献 ${ }^{14}$ 研究的 $\mathrm{La}_{1.6} \mathrm{Sr}_{0.4} \mathrm{NiO}_{4}$ 阴极的极化 面电阻相比数值更小, 这可能是由于 $\mathrm{Cu}$ 离子的掺杂 增加了材料的氧空位浓度, 改善了电极的电催化活 性, 使得电极的极化电阻数值有所下降. 类似的结 果在 $\mathrm{Ba}_{0.5} \mathrm{Sr}_{0.5} \mathrm{Fe}_{1-x} \mathrm{Cu}_{x} \mathrm{O}_{3}$ 阴极材料中也有报道. ${ }^{23}$ 接下 来的研究中, 以最佳条件制得的 $\mathrm{La}_{1.6} \mathrm{Sr}_{0.4} \mathrm{Ni}_{0.4} \mathrm{Cu}_{0.6} \mathrm{O}_{4}$ 电极为研究对象.

为了明确电极上的反应动力学过程, 研究了电 极的极化电阻随测试温度和氧分压的变化情况. 图 5 为 $\mathrm{La}_{1.6} \mathrm{Sr}_{0.4} \mathrm{Ni}_{0.4} \mathrm{Cu}_{0.6} \mathrm{O}_{4}$ 电极在 $650{ }^{\circ} \mathrm{C}$ 不同氧分压下 的交流阻抗谱. 从图中可以观察到, 随着氧分压的 增大, 电极的极化电阻 $R_{\mathrm{p}}$ 逐渐降低, 并且在低频和 高频段出现两个半圆弧; 当氧分压发生变化时, 低 频段的圆弧变化比较大, 而高频段变化很小, 低频

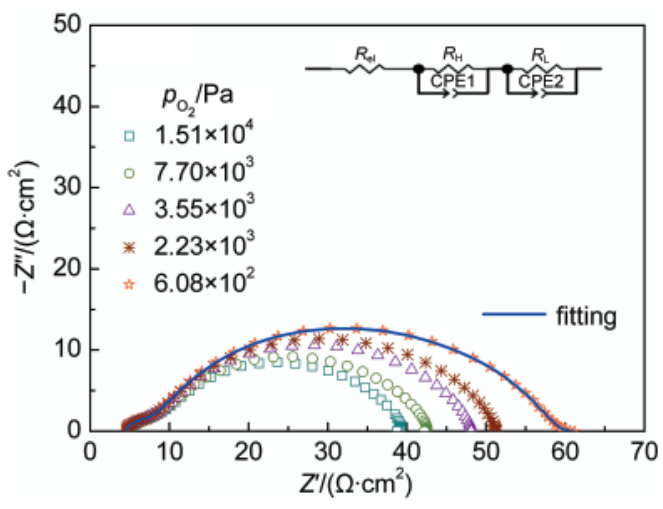

图 $5650{ }^{\circ} \mathrm{C}$ 时 $\mathrm{La}_{1.6} \mathrm{Sr}_{0.4} \mathrm{Ni}_{0.4} \mathrm{Cu}_{0.6} \mathrm{O}_{4}$ 电极在 不同氧分压下的阻抗谱

Fig.5 Impedance spectra of $\mathrm{La}_{1.6} \mathrm{Sr}_{0.4} \mathrm{Ni}_{0.4} \mathrm{Cu}_{0.6} \mathrm{O}_{4}$ electrode at $650{ }^{\circ} \mathrm{C}$ under various oxygen partial pressures Inset shows the equivalent circuit. $R_{\mathrm{cl}}$ is the resistance of the electrolyte and lead wires, $R_{\mathrm{H}}$ and $R_{\mathrm{L}}$ are the resistances corresponding to the high frequency arc and low frequency arc, and CPE1 and CPE2 are the two constant phase elements.

弧电阻大于高频弧电阻. 用图 5 内插图所示的等效 电路对阻抗谱进行拟合, 图中 $R_{\mathrm{el}}$ 表示由 $\mathrm{CGO}$ 电解 质中氧离子传递引起的阻抗贡献, CPE1 和 CPE2 为 两个恒定相元, 通过拟合可以得到不同氧分压下高 频电阻值 $\left(R_{\mathrm{H}}\right)$ 和低频电阻值 $\left(R_{\mathrm{L}}\right)$. 另外, 由阻抗谱的 高频和低频端的两个半圆弧的弛豫频率和阻抗数 值, 分别计算得到它们对应的特征电容值为 $10^{-5}$ 和 $10^{-3} \mathrm{~F}$, 表明这两个半圆弧是电极上两个不同的电化 学反应过程所引起的 ${ }^{24}$ 当氧分压发生变化时, 高频 弧和低频弧的特征电容值不发生变化. 利用极化电 阻在不同氧分压下的数值, 得到了在 $650^{\circ} \mathrm{C}$ 测试条 件下 $\mathrm{La}_{1.6} \mathrm{Sr}_{0.4} \mathrm{Ni}_{0.4} \mathrm{Cu}_{0.6} \mathrm{O}_{4}$ 电极的极化电阻 $R_{\mathrm{p}}$ 随氧分压 的变化曲线, 如图6所示. 由图可以看出, 极化电阻 $R_{\mathrm{p}}$ 随着氧分压的增大而减小. 极化电阻随氧分压的 变化可以通过下式来描述: $R_{\mathrm{p}}=R_{0}\left(p_{\mathrm{O}_{2}}\right)^{-n}, n$ 的数值反 映了电极上所发生的电化学反应的类型. ${ }^{25,26}$

$$
n=1 / 2, \mathrm{O}_{2, \text { ads }} \rightleftharpoons 2 \mathrm{O}_{\text {ads }}
$$




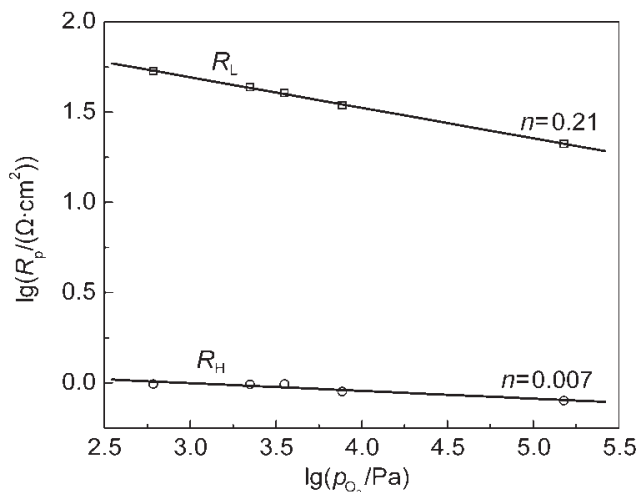

图 $66^{650}{ }^{\circ} \mathrm{C}$ 温度下 $\mathrm{La}_{1.0} \mathrm{Sr}_{0.4} \mathrm{Ni}_{0.4} \mathrm{Cu}_{0.6} \mathrm{O}_{4}$ 电极极化电阻 $\left(R_{\mathrm{p}}\right)$ 随氧分压的变化曲线

Fig.6 Polarization resistance $\left(R_{\mathrm{p}}\right)$ of $\mathrm{La}_{1.6} \mathrm{Sr}_{0.4} \mathrm{Ni}_{0.4} \mathrm{Cu}_{0.6} \mathrm{O}_{4}$ electrode $v s p_{\mathrm{O}_{2}}$ at $650^{\circ} \mathrm{C}$

$n=1 / 4, \mathrm{O}_{\text {ads }}+2 \mathrm{e}^{\prime}+\mathrm{V}_{o}^{* \bullet} \rightleftharpoons \mathrm{O}_{0}^{x}$

$n=0, \mathrm{O}_{\mathrm{TPB}}^{2-}+\mathrm{V}_{\circ}^{* \bullet} \rightleftharpoons \mathrm{O}_{\mathrm{O}}^{x}$

由图 6 可以看出, $R_{\mathrm{L}}$ 随着氧分压的增大而减小, 而 $R_{\mathrm{H}}$ 随着氧分压的增大基本不发生变化. 根据极化 电阻与氧分压关系式从图 6 可以得到 $R_{\mathrm{H}}$ 和 $R_{\mathrm{L}}$ 对应 的 $n$ 值分别为 0.007 和 0.21 . 这样可以判断 $R_{\mathrm{H}}$ 所代表 的是电极上发生的氧离子从三相界面向电解质 $\mathrm{CGO}$ 转移的过程, 而 $R_{\mathrm{L}}$ 则对应于电极上电荷的迁移 过程. ${ }^{27}$ 此外, 在测试温度和氧分压范围内, $R_{\mathrm{L}}$ 的数 值均大于 $R_{\mathrm{H}}$, 这表明电极上电化学反应的速率控制 步骤为电荷的迁移过程.

在电池实际工作时电极电位会偏离平衡电位, 这种现象称为电极的极化, 偏离的电压称为电极的 过电位, 并且过电位的高低可以直接影响到电池的 输出特性与指标. 本文采用电压阶梯扫描法, 测试 了 $\mathrm{La}_{1.6} \mathrm{Sr}_{0.4} \mathrm{Ni}_{0.4} \mathrm{Cu}_{0.6} \mathrm{O}_{4}$ 电极的阴极极化性能. 图 7 为

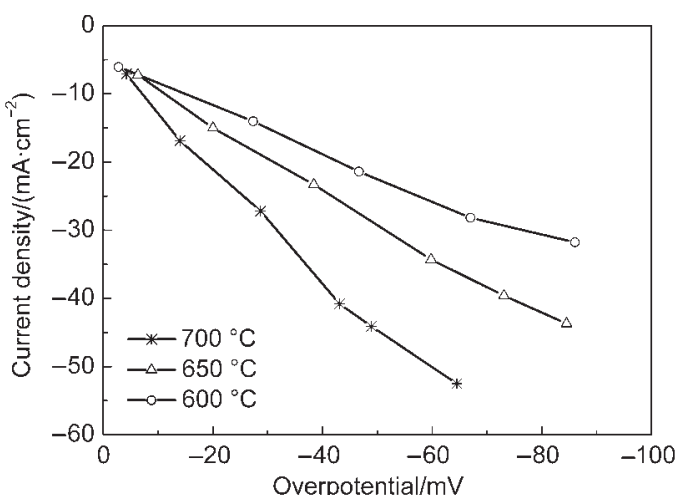

图 $7 \mathrm{La}_{1.6} \mathrm{Sr}_{0.4} \mathrm{Ni}_{0.4} \mathrm{Cu}_{0.6} \mathrm{O}_{4}$ 电极在空气中不同温度下的 电流密度-过电位曲线

Fig.7 Current density-overpotential curves for $\mathrm{La}_{1.6} \mathrm{Sr}_{0.4} \mathrm{Ni}_{0.4} \mathrm{Cu}_{0.6} \mathrm{O}_{4}$ electrode measured in air at various temperatures
$\mathrm{La}_{1.6} \mathrm{Sr}_{0.4} \mathrm{Ni}_{0.4} \mathrm{Cu}_{0.6} \mathrm{O}_{4}$ 电极在空气中不同温度下测量得 到的过阴极电流密度-电位曲线, 由图可以看出, 在 相同的电流密度下随着测试温度的升高, $\mathrm{La}_{1.6} \mathrm{Sr}_{0.4} \mathrm{Ni}_{0.4} \mathrm{Cu}_{0.6} \mathrm{O}_{4}$ 的阴极过电位逐渐降低. 在 $700^{\circ} \mathrm{C}$ 下, 过电位较低的弱极化区 $(<50 \mathrm{mV})$, 当阴极过电 位为 $45 \mathrm{mV}$ 时, 阴极电流密度为 $45 \mathrm{~mA} \cdot \mathrm{cm}^{-2}$. 这一 结果优于同温度下 $\mathrm{La}_{2} \mathrm{NiO}_{4}$ 阴极材料测试结果, ${ }^{28}$ 这 说明该电极具有较好的电催化性能, $\mathrm{La}_{1.6} \mathrm{Sr}_{0.4} \mathrm{Ni}_{1-x} \mathrm{Cu}_{x} \mathrm{O}_{4}$ 是一种潜在的固体氧化物燃料电 催化阴极材料.

\section{4 结 论}

采用甘氨酸-硝酸盐法合成了 $\mathrm{La}_{1.6} \mathrm{Sr}_{0.4} \mathrm{Ni}_{1-x} \mathrm{Cu}_{x} \mathrm{O}_{4}$ 阴极材料. 在 $1000{ }^{\circ} \mathrm{C}$ 空气中烧结得到的电极与 $\mathrm{CGO}$ 电解质可形成良好的接触界面, 其中当 $\mathrm{Cu}$ 掺 杂量为 0.6 时, 电极 $\mathrm{La}_{1.6} \mathrm{Sr}_{0.4} \mathrm{Ni}_{0.4} \mathrm{Cu}_{0.6} \mathrm{O}_{4}$ 的极化电阻值 最小, 在 $750{ }^{\circ} \mathrm{C}$ 时为 $0.35 \Omega \cdot \mathrm{cm}^{2}$. 氧分压测试结果显 示, 阴极反应的速率控制步骤主要为电荷的迁移过 程. 在 $700{ }^{\circ} \mathrm{C}$ 的测试条件下, 当 $\mathrm{La}_{1.6} \mathrm{Sr}_{0.4} \mathrm{Ni}_{0.4} \mathrm{Cu}_{0.6} \mathrm{O}_{4}$ 电 极阴极过电位为 $45 \mathrm{mV}$ 时, 电流密度达 $45 \mathrm{~mA} \cdot \mathrm{cm}^{-2}$.

\section{References}

(1) Skinner, S. J.; Kilner, J. A. Solid State Ionics 2000, 135, 709. doi: 10.1016/S0167-2738(00)00388-X

(2) Ding, X. F.; Kong, X.; Jiang, J. G.; Cui, C. Int. J. Hydrog. Energy 2009, 34, 6869. doi: 10.1016/j.ijhydene.2009.06.041

(3) Peng, B.; Chen, G.; Wang, T.; Zhou, J.; Guo, J. J.; Cheng, Y. H.; Wu, K. J. Power Sources 2012, 201, 174.

(4) Taguchi, H. Mater. Res. Bull. 2001, 36, 1361. doi: 10.1016/ S0025-5408(01)00631-6

(5) Vashook, V.; Girdauskaite, E.; Zosel, J.; Wen, T. L.; Ullmann, H.; Guth, U. Solid State Ionics 2006, 177, 1163. doi: 10.1016/j. ssi. 2006.05 .018

(6) Minervini, L.; Grimes, R. W.; Kilner, J. A.; Sickafus, K. E. J. Mater. Chem. 2000, 10, 2349. doi: 10.1039/b004212i

(7) Yang, X. M.; Luo, L. T.; Zhong, H. Appl. Catal. A 2001, 207, 69. doi: 10.1016/S0926-860X(00)00658-X

(8) Bochkov, D. M.; Kharton, V. V.; Kovalevsky, A. V.; Viskup, A. P.; Naumovich, E. N. Solid State Ionics 1999, 120, 281. doi: 10.1016/S0167-2738(99)00019-3

(9) Vashooka, V. V.; Trofimenko, N. E.; Ullmann, H.; Makhnach, L. V. Solid State Ionics 2000, 131, 329. doi: 10.1016/S0167-2738 (00)00571-3

(10) Yang, J. F.; Cheng, J. G.; Fan, Y. M.; Wang, R.; Gao, J. F. Acta Phys. -Chim. Sin. 2012, 28, 1230. [杨俊芳, 程继贵, 焚玉萌, 王＼cjkstart睿, 高建峰.物理化学学报, 2012, 28, 1230.] doi: 10.3866/ PKU.WHXB201202232 
(11) Khandale, A. P.; Bhoga, S. S. J. Power Sources 2010, 195, 7974. doi: 10.1016/j.jpowsour.2010.06.044

(12) Al Daroukh, M.; Vashook, V. V.; Ullmann, H.; Tietz, F.; Arual Raj, I. Solid State Ionics 2003, 158, 141. doi: 10.1016/ S0167-2738(02)00773-7

(13) Jorgensen, J.; Dabrowski, B.; Pei, S.; Richards, D. R.; Hinks, D. G. Phys. Rev. B 1989, 40, 2187. doi: 10.1103/PhysRevB.40.2187

(14) Li, Q.; Fan, Y.; Zhao, H.; Huo, L. H. Chin. J. Inorg. Chem. 2006, 22, 2025. [李 强, 范 勇, 赵 辉, 霍丽华. 无机化学 学报, 2006, 22, 2025.]

(15) Inprasit, T.; Limthongkul, P.; Wongkasemjit, S. ECS Transactions 2009, 25, 2581 .

(16) Ling, Y. H.; Zhao, L.; Lin, B.; Dong, Y. C.; Zhang, X. Z.; Meng, G. Y.; Liu, X. Q. Int. J. Hydrog. Energy 2010, 35, 6905. doi: 10.1016/j.ijhydene.2010.04.021

(17) Zhang, H.; Wang, T.; Dong, X.; Lin, W. J. Nat. Gas Chem. 2009, 18, 45. doi: 10.1016/S1003-9953(08)60084-5

(18) Zha, S. W.; Moore, A.; Abernathy, H.; Liu, M. L. J. Electrochem. Soc. 2004, 151, A1128.

(19) Zhao, H.; Li, Q.; Sun, L. P. Sci. China Ser. B-Chem. 2011, 54, 898. doi: 10.1007/s11426-011-4290-2
(20) Aguadero, A.; Alonso, J. A.; Escudero, M. J.; Daza, L. Solid State Ionics 2008, 151, 393.

(21) Li, Q.; Zhao, H.; Huo, L. H.; Sun, L. P.; Cheng, X. L.; Grenier, J. C. Electrochem. Commun. 2007, 9, 1508. doi: 10.1016/ j.elecom.2007.02.013

(22) Li, Q.; Fan, Y.; Zhao, H.; Sun, L. P.; Huo, L. H. J. Power Sources 2007, 167, 64. doi: 10.1016/j.jpowsour.2007.02.018

(23) Efimov, K.; Halfer, T.; Kuhn, A.; Heitjans, P.; Caro, J.; Feldhoff, A. Chem. Mater 2010, 22, 1540. doi: 10.1021/cm902882s

(24) Irvine, J. T. S.; Sinclair, D. C.; West, A. R. Adv. Mater. 1990, 2 , 132. doi: 10.1002/adma.19900020304

(25) Takeda, Y.; Kanno, R.; Noda, M.; Yamamoto, O. J. Electrochem. Soc. 1987, 134, A2656.

(26) Sicbeit, E.; Hammouche, A.; Kleitz, M. Electrochim. Acta 1995, 40, 1741. doi: 10.1016/0013-4686(94)00361-4

(27) Li, Q.; Zeng, X.; Sun, L. P.; Zhao, H.; Huo, L. H.; Grenier, J. C. Int. J. Hydrog. Energy 2012, 37, 2552. doi: 10.1016/j.ijhydene. 2011.11.014

(28) Mauvy, F.; Lalanne, C.; Bassat, J. M.; Grenier, J. C.; Zhao, H.; Dordor, P.; Stevens, P. J. Eur. Ceram. Soc. 2005, 25, 2669. doi: 10.1016/j.jeurceramsoc.2005.03.120 